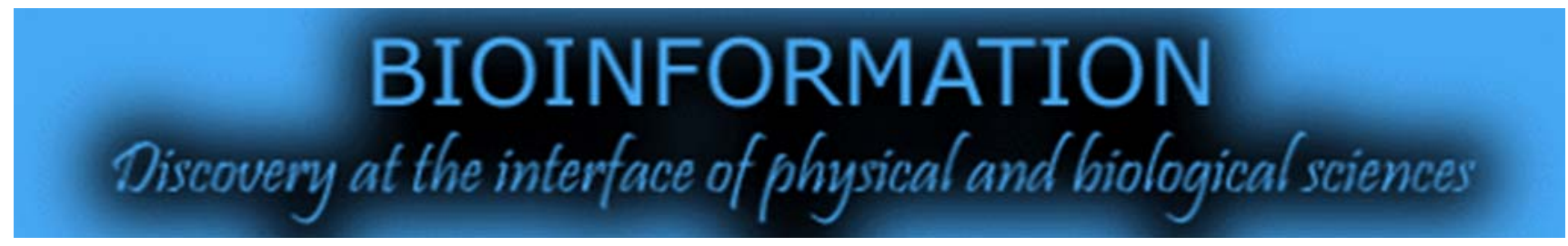

\title{
Immune surveillance of nasopharyngeal carcinoma (NpC)
}

\author{
Oluwadayo Oluwadara1,4, Andre Barkhordarian ${ }^{1,4}$, Luca Giacomelli2,4, Xenia Brant ${ }^{1,3,4}$, Francesco \\ Chiappelli ${ }^{1,4 *}$
}

${ }^{1}$ Oral Biology \& Medicine, School of Dentistry, UCLA; ${ }^{2}$ Istituto di Stomatologia, Lido di Camaiore, Italy; ${ }^{3}$ CEO IPSEMG, School of Dentistry, Belo Horizonte, Brazil; ${ }^{4}$ Evidence-Based Decisions Practice-Based Research Network (ebd-pbrn.org) and Div Biology \& Medicine, CHS 63-090, UCLA School of Dentistry, Los Angeles, CA 90095-1668; Francesco Chiappelli - Email: chiappelli@dentistry.ucla.edu; *Corresponding authors

Received October 10, 2011; Accepted October 11, 2011; Published October 31, 2011

\begin{abstract}
:
In the U.S., nasopharyngeal carcinoma ( $\mathrm{NpC}$ ) kills $>7,600$ each year. Deaths are predominantly among adult men, and in most cases, early detection and treatment can save lives. Despite the annual spending of approximately 3.2 billion dollars on head and neck cancer research, $\mathrm{NpC}$ remains a neglected disease since its fatality rates are among the lowest nation wide. The relative survival rates from $\mathrm{NpC}$ have not improved in the U.S. in the last 20 years. Infection with Epstein Barr Virus (EBV) is an important co-factor in the etiology of NpC. In other regions of the word (e.g., South-East Asia, Latin America), EBV infection and NpCrelated prevalence and mortality are substantially higher and more alarming. Epidemiological data indicate high prevalence of EBV infection and increased risk for $\mathrm{NpC}$ among Central and South American and Asian immigrants in the U.S., and also predict a sharp increase in $\mathrm{NpC}$ incidence in the next decade. To face this emerging threat, it is important to develop and validate novel modes of detection and intervention for $\mathrm{NpC}$. To this end, we characterized the proteomic signature of $\mathrm{NpC}$, and of the tumor infiltrating lymphocytes of the $\mathrm{CD} 8+$, activated $(\mathrm{CD} 38+, \mathrm{mTOR}+)$ and regulatory immune cell (FoxP3+) phenotype. Paraffinized biopsies were processed, and tissue microarrays constructed and tested by immunohistochemistry and triimmunohistofluorescence for a battery of signaling markers, including AKT and PI3K, in conjunction with EBV status and ANKRD11, an NpC susceptibility biomarker. Microphotographs, analyzed and quantified by confocal microscopy and fractal analysis, suggest new avenues for immunotherapies of $\mathrm{NpC}$.
\end{abstract}

Keywords: Nasopharyngeal carcinoma, Epstein Barr Virus, Tumor Infiltrating Lymphocytes, tissue microarray, immunohistochemistry, immunohistofluorescence, fractal analysis, proteomic signature, signaling pathways, AKT, Pi3K, mTOR, CD8+, CD38+, FoxP3, ANKRD11

\section{Background:}

Nasopharyngeal carcinoma $(\mathrm{NpC})$ is a malignant tumor of epidermoid origin, which occurs mostly in the lateral wall of the lympho-epithelium of the nasopharnyx. NpC has a geographical distribution, being most common among adults in Latin America and Southeastern Asia (prevalence: 15$50 / 100,000)$, and African children. Prevalence of NpC is 2:1 higher in males, compared to females. Increased risk for $\mathrm{NpC}$ is associated with HLA-2, HLA-B17, and HLA-Bw26 haplotypes, and include other etiological factors (e.g., environment, diet, and Epstein-Barr virus infection [EBV; human herpesvirus-4, HHV-4, orally transmitted $\gamma-1$ herpesvirus]). Exposure to cigarette smoking and other sources of nitrosamine as well as to polycyclic hydrocarbons also increase risk for $\mathrm{NpC}$. Due to its rather hidden anatomical location, $\mathrm{NpC}$ is often difficult to detect early and is commonly diagnosed at an advanced stage, which curtails survival [1-3]. 
The NpC-susceptibility protein and medullo-blastoma antigen, Ankyrin repeat domain 11 (ANKRD11, aka ANR11 or ANCO1), is a p53-interacting protein that enhances the activity of p53. As a member of the family of ankyrin repeats containing cofactors (ANCOs), ANKRD11 interact with p160 co-activators to inhibit ligand-dependent transactivation. ANKRD11 enhances the DNA-binding properties of mutant p53 (R273H) to the CDKN1A promoter, thus mediating the restoration of normal p53 function in some cancer-related p53 mutations [4].

The identification of the proteomic signature of $\mathrm{NpC}$, and tumor infiltrating lymphocyte populations (TILs) could ensure novel modes of detection and intervention. This study sought to define and to characterize biomarkers of activated signaling pathways, and of the populations of TILs in NpC tissue microarrays. Immunohistochemistry (IHC) and immunohistofluorescence (IHF) imaging profiles of certain salient such markers converge to provide a novel perspective on the cellular immune surveillance in $\mathrm{NpC}$.

We tested the PI3K/AKT/mTOR pathway because AKT (aka AKT1 of the protein kinase B [PKB] family) promotes several types of cancer by acting as an anti-apoptotic factor. AKT is activated through phosphatidylinositol 3-kinase (PI3K), and is critical for transmitting growth promoting signals, in large part, but not exclusively, by activating the mammalian target of rapamycin (mTOR), a regulator of cell growth, proliferation, motility, and survival $[5,6]$. The PI3K/AKTmTOR pathway is often overactive in cancer, and favors cell division while reducing apoptosis, and has become the target of many efforts toward proteomics-based therapies [7].

We tested for the invasion of $\mathrm{CD} 8+\mathrm{T}$ cells, which are the principal population of TIL-Ts in several carcinomas, although the CD8 phenotypic cell surface marker is also displayed on natural killer and dendritic cells. Because TIL's-T are activated effector cells, we monitored cells that expressed the marker of activation CD38, the multifunctional ectoenzyme cyclic ADP ribose hydrolase, which catalyzes the synthesis and hydrolysis of cyclic ADP-ribose (cADPR), a regulator of $\mathrm{Ca}^{++}$flux. The loss of CD38 expression and function in CD8+ cells is associated with impaired immune responses, metabolic disturbances, and behavioral modifications. Activated $\mathrm{B}$ cells can also express CD38, and CD38+ B lymphocytes that express surface IgD, while remaining IgM-, can be selectively derived from human nasopharynx-associated lymphoid tissue. These activated B cells give rise to extrafollicular IgD+ plasma cells, which however remain stunted for downstream immunoglobulin class switch and cell maturation [8-10]. Infection with EBV establishes a lytic infection in the oropharynx and a contemporaneous latent infection into the B-lymphoid population with extrafollicular memory CD38+ B cells [10].

TILs-B constitutes a newly identified aspect of the immune surveillance mechanisms for a variety of malignancies. TILs-B, which account for $30-40 \%$ of the TILs, generally show evidence of maturation and recognize specific tumor and self-antigens. The presence of TILs-B yields a favorable outcome for tumor immune surveillance because they functionally associate closely with TILs-T and other tumor immune surveilling cell populations. In brief, and perhaps particularly in the instance of EBV infection of the nasopharyngeal epithelium, we propose that infiltrating activated B cells might play an important role to enhance $\mathrm{T}$ cell responses by producing specific antibody populations, by secreting stimulatory cytokines and chemokines, by serving as local antigen-presenting cells, and by organizing and sustaining the formation of tertiary lymphoid structures that can sustain long-term cellular immunity [11].

We also assessed regulatory immune cells expressing forkhead box P3 (FoxP3+) among CD8+ and CD38+ TILs, because experimental induction or administration of regulatory $\mathrm{T}$ cells (Tregs, CD4+/CD8+FoxP3+) can lead to marked reductions in cellular immunity, suggesting that cellular therapies targeting Tregs could help overcome diseases where immunotoxicity against certain targets, such as cancer, need to be maximized [12]. Regulatory B cells (Bregs) have now been identified, which potently influences the induction, activation and maintenance of function of T effector cells [13].

\section{Methodology:}

Paraffinized biopsy specimens of $\mathrm{NpC}$ tissue arrays were purchased from Pantomics, Inc pantomics.com; NPC961 Nasopharyngeal carcinoma tissue array, 96 cores, $1.1 \mathrm{~mm}, 4$ $\mathrm{mm}$ ). The micro-arrays contained 31 cases of primary tumors with paired normal tissues, 17 of lymph node metastatic tumors and 12 of normal/reactive nasopharyngeal mucosa. Each core was obtained from surgical resection, and fixed in 10\% neutral buffered formalin for 24 hours before mounting. Triplicates of each set of four TMAs with 96 biopsies per slide were used for immunohistochemistry experiments, as described previously [14-16].

For IHC, primary antibodies were from Abcam (abcam.com). Mouse anti-human Epstein Barr Virus (EBV) Early Antigen Diffuse and Latent Membrane Protein were used at a dilution of 1:100. Mouse anti-human EBV Nuclear Antigen was used at a dilution of 1:50. Rabbit anti-human AKT and anti-human Pi3K proteins were used at the dilution of 1:50. Rabbit anti-human mTOR protein was used at the dilution of 1:250. Biotinylated secondary goat anti-rabbit antibody was used with the Vectastatin avidin-biotin complex (ABC) kit (VectorLaboratories, vectorlabs.com). Color was developed with the Vector Nova Red Substrate kit. For IHF, cell nuclei were stained with 4',6-diamidino-2-phenylindole (DAPI), a fluorescent stain that binds strongly to A-T rich regions of DNA (emission maximum: 461nm, blue visible region). Mouse monoclonal anti-human anti-CD8 antibody conjugated to fluorescein (Becton Dickinson, bd.com) (emission maximum: 521nm: green visible region), and mouse monoclonal antihuman anti-CD38 antibody conjugated to rhodamine (Becton Dickinson) (emission maximum: 545nm, red visible region) were used. The FoxP3 rabbit anti-human antibody was from abcam, and it was used with a fluorochrome-conjugated antirabbit goat IgG obtained from Sigma.

As previously described [16], slides were scanned at 40x magnification. The Aperio Imagescope software (Version 9.1.19.1569) was used for scoring, additional analysis and subsequent magnification. Two independent assessors evaluated the expression (signals) of each epitope based on the nuclear and cytoplasmic stains. A circle of tissue on the TMA was scored from 0 to 100 , based on the extent of stain signals over the tissue. A score of 50 was interpreted as staining covering half of a circle of tissue, score of 75 means $3 / 4$ of the 
tissue were stained, while a score of 10 simply covers a small arc of a circle of tissue. Expressions of markers were ranked as low (signal scores: 1-24), negative (signal scores: 0-10), and high (signal scores $\geq 25$ ). Expression level of each EBV marker was assigned a score of +1 . Pooled results for each sample gave a score of either +1 (EBV negative or very low), +2 (intermediate $\mathrm{EBV}),+3$ (intense staining for EBV markers),+4 (strong positive signal for all three EBV markers).
Microphotographs were obtained at 200X or $400 \mathrm{X}$ magnifications with a Nikon microscope with bright phase for IHC, and with fluorescent illumination for IHF. Confocal microscopy was also obtained with an Olympus microscope. Statistical evaluation $(\alpha=0.05)$ of the effectiveness of molecular markers of EBV as adjunctive techniques for detection of oral premalignant and malignant $\mathrm{NpC}$ lesions was obtained as previously described $[\mathbf{1 6 , 1 7 ]}$. Fractal analysis was as previously described $[\mathbf{1 4}, \mathbf{1 5}]$.
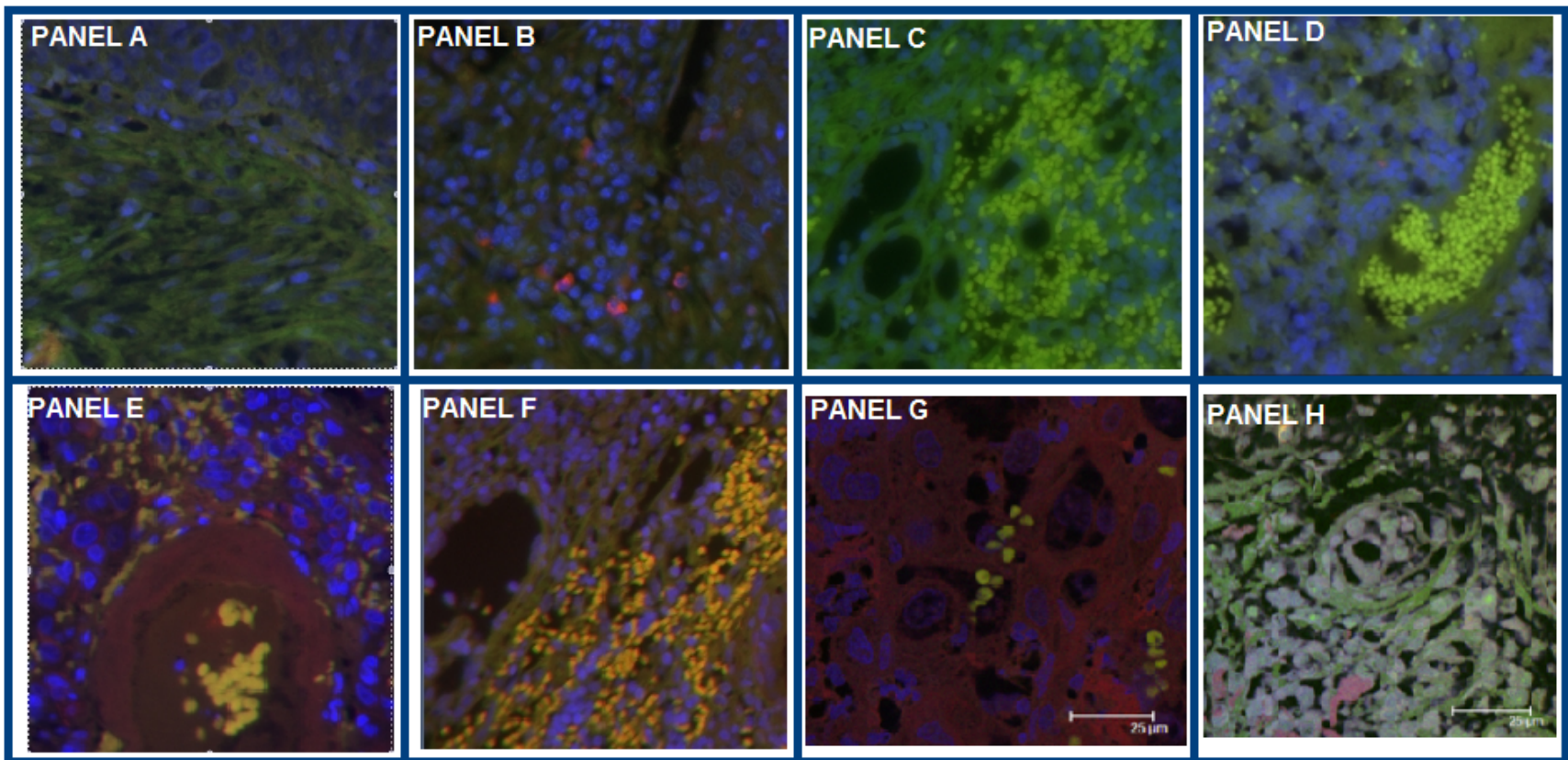

Figure 1: Pantomics, NPC961 microarrays were immunostained with fluorescent probes, and microphotography obtained at 200X or 400X. Fields show the three superimposed fluorochromes: the blue DAPI stain shows the cell nuclei; the green stain represent the immunofluorescence contributed by anti-CD38 fluorescein; the red stain corresponds to rhodamine immunostaining of the probant antigen; the whitish/yellowish-light green stain corresponds the overlap of the fluorescien and rhodamine stains. Panel A confirms virtually complete overlap of NpC susceptibility antigen, ANKRD11, with cells expressing the activation marker CD38. Panel B shows that the CD8+, rhodamine-conjugated, cells hardly overlap with the CD8+ cells, which is confirmed by confocal microscopy (Panel G), with purposeful reversal of the fluorochromes. Panel C and D show the disparate localization of CD38+FoxP3+ invading lymphocytes in NpC lesions proper (Panel C), compared to tissue taken adjacent to NpC tumor lesions (Panel D). Panels E and F confirm the distinct localization of immigrating CD38+ lymphocytes in tissue adjacent to NpC lesions (Panel E), compared to NpC lesions proper (Panel F). Panels E and F show activated TILs expressing mTOR. Panel H shows by confocal microscopy a high level of Akt expression pervasive in lymphocytes still captured in the blood vessels of NpC lesions proper, as well as in the tissue parenchyma

\section{Discussion:}

Early experiments established the EBV status of the NpC microarrays. EBV staining is punctate, and was optimized by using three distinct antibodies against the epitopes for latent membrane protein expression of the early EBV antigen, for diffuse expression, and for expression of the nuclear EBV antigen. Samples with more definite reactivity with all three EBV markers were ranked EBV4+, and samples with the lesser reactivity with barely one of the EBV antigens tested were ranked EBV+1. The differences in EBV immunoreactivity appeared directly related to the functional activity of the cells, as determined by fractal analysis (Benoit fractal analysis,

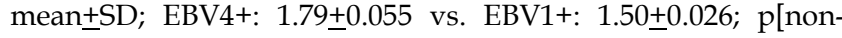
matched, equal sample size $t$ test $]=0.00005$.

Cellular functional activity was taken as the state of activation and transmembrane signaling. Scoring ranked immunoreactivity to Akt and PI3K, which are directly upstream ISSN 0973-2063 (online) 0973-8894 (print)

Bioinformation 7(5):271-275 (2011) to mTOR, and for mTOR as well. NpC samples with low to moderate EBV infection had lower PI3K staining, compared to EBV4+ tissues (median score across independent evaluators, EBV4+: 13.2 vs. EBV3+: 10.5 vs. EBV2+: 5.9 vs. EBV1+: 9.4). Similar trends were obtained with Akt immunostaining (median score, EBV4+: 50.7 vs. EBV3+: 42.9 vs. EBV2+: 24.4 vs. EBV1+: 8.9) and with mTOR immunostaining (median score, EBV4+: 45.6 vs. EBV3+: 33.8 vs. EBV2+: 29.1 vs. EBV1+: 11.7). Statistical analysis of these scores was rendered difficult due to the large amount of random error. However, Wilcoxon analyses disclosed a difference in scores of Akt and mTOR immunoreactivities between low and moderate EBV infection $(\mathrm{EBV}+1-\mathrm{EBV}+3)$, compared to $\mathrm{EBV}+4\left(\mathrm{p}=0.0006\right.$ and $\mathrm{p}<1.7 \mathrm{X} 10^{-9}$, respectively). Akt and mTOR immunostaining was strongly correlated, revealing as much as $45 \%$ shared variance. 
Fractal analyses confirmed the interdependent nature of the relationship among PI3K, Akt and mTOR, with respect to cell dimension, and presumably active cell growth. PI3K and Akt immunostained EBV4+ were found to be overall larger, and presumably more metabolically active than EBV1+ cells (respectively, PI3K fractal comparison - EBV4+: 1.43+0.03 vs. EBV1+: 1.34 $\pm 0.02 ; \mathrm{p}=0.0009$; Akt fractal comparison - EBV4+: $1.56 \pm 0.08$ vs. EBV1+: $1.42 \pm 0.03$; t test $\mathrm{p}=0.01295)$. By contrast, mTOR immunostained EBV4+ were not found to be significantly larger than EBV1+ cells (EBV4+: $1.47 \pm 0.12$ vs. EBV1+: 1.38+0.019; $\mathrm{p}=0.11$ ), an outcome more likely due to the large random error in measurement rather than to the lack of a biological effect.

Taken together, our IHC data could signify important deregulation in the mTOR pathway in $\mathrm{EBV}+$ samples of $\mathrm{NpC}$ biopsies, reflected in part if alteration of the upstream regulatory activities of $\mathrm{PI} 3 \mathrm{~K}$ and Akt. The observations established a direct correspondence between the activation of these metabolic pathways and the intensity of EBV infection, as defined by the intensity of staining of three distinct EBV epitopes.

A second round of experiments sought to characterize which cell populations was mTOR-activated in these NpC samples. Tri-fluorescence IHF experiments confirmed complete overlap of cells immunostained for the $\mathrm{NpC}$ susceptibility antigen, ANKRD11 [4], and for the ectoenzyme cyclic ADP ribose hydrolase, CD38, a common marker of lymphocyte activation [10] (Figure 1, panel A). This important control ascertained the histologic nature of the $\mathrm{NpC}$ biopsies, which were expected to be of lympho-epithelial origin in the nasopharynx. By contrast, there appears to be relatively few TILs-T of the CD38 phenotype (Figure 1, panel B), and these invading CD8+ do not express the activation marker CD38. Panel C in Figure 1 shows, nevertheless, that there is a substantial influx of immune cells in the $\mathrm{NpC}$ lesions, and that these infiltrating lymphocytes express both the CD38 activation marker, and the marker of regulatory immune cells, FoxP3, indicating a significant migration of Bregs in NpC. This observation is confirmed at lower magnification in Panel D, which shows the substantial accumulation of CD38+FoxP3+ cells within the blood vessels of $\mathrm{NpC}$ lesions. It is quite evident from panels E \& F (Figure 1) that these migrating CD38+ lymphocytes correspond to the mTOR+ cells analyzed by fractal analysis. Noteworthy, migrating CD38+ lymphocytes are primarily viewed in the blood vessels of tissue samples taken adjacent to tumorigenic NpC (Figure 1, panels D $\&$ E), but are localized in the tissue parenchyma of the $\mathrm{NpC}$ lesions proper (Figure 1, panels C \& F).

We utilized confocal microscopy with reversed fluorochromes to verify our observations. Panel G of Figure 1 confirms the relative paucity of invading $\mathrm{CD} 8+$ cells in NpC. Furthermore, Panel $\mathrm{H}$ indicates that TILs in $\mathrm{NpC}$ found in the blood vessels or the tissue parenchyma are activated and express, in this present case, high levels of Akt, upstream of the activation of mTOR (Figure 1, panels E \& F).

\section{Conclusion:}

Histologically, the nasopharyngeal tissue is lympho-epithelial, rich of resident and transient $\mathrm{B}$ cells. This confers a distinct and unique micro-environment for carcinomas [18, 19] predicated primarily on the immunoregulatory role of B cells.

Our data confirm that $\mathrm{NpC}$, and adjacent areas, are endowed with relatively few TIL's with CD8+ T phenotype. Rather, NpC parenchyma exhibit substantial infiltration by TILs that express the marker of regulatory lymphocytes, FoxP3 [20], the marker of activation (CD38), and markers of transmembrane and transcytosolic signaling (PI3K, Akt, mTOR pathway). These observations suggest that the predominant immune surveillance mechanism for controlling $\mathrm{NpC}$ progression is mediated by the regulatory role of resident plasma B cell population, and Bregs.

Our data also confirm that CD38-mediated signal transduction in B cells induces activation of the PI3K pathway [21]. In line with recent findings that $\mathrm{mTOR}$ activation in B cells leads to a significant block in further B cell maturation [22], our results suggest that the proteomic signature of TILs-B in NpC may determine timely and critical interwoven translational research and interventions [23] aimed at redirecting B cell activation pathways in $\mathrm{NpC}$ immune surveillance.

Recent evidence supports this working model by suggesting that Akt emits two mutually counter-balancing signals in regulating $\mathrm{B}$ cell differentiation and apoptosis: one involves mTOR activation, and the other remains to be elucidated [22]. Therefore, our data may not be an epiphenomenon, but rather suggest that the FoxP3+ Bregs population might play an important role in modulating the tumor microenvironment [19] of $\mathrm{NpC}$, in a manner akin to other B cell-related immunemodulatory events [20].

\section{Acknowledgement:}

We warmly thank the Evidence-Based Decisions Active Groups of Stakeholders (EBD-AGS) of the EBD-Practice-Based Research Network and the EBD Study Group for the invaluable critical contributions to this work. We also thank the UCLA Senate for funding to FC, and the undergraduate students who participated in these studies, Raisa Avezova and David Jourabhi.

\section{References:}

[1] Sugerman PB et al. Crit Rev Oral Biol Med. 2002 13: 350 [PMID: 12191961]

[2] Young LS \& Rickinson AB, Nat Rev Cancer. 2004 4: 757 [PMID: 15510157]

[3] Oluwadara OO \& Chiappelli F, Bioinformation 2009 3: 332 [PMID: 19707295]

[4] Neilsen PM et al. J Cell Sci. 2008 121: 3541 [PMID: 18840648]

[5] Hay N \& Sonenberg N, Genes Dev. 2004 18:1926 [PMID: 15314020]

[6] Sarbassov DD et al. Science. 2005 307: 1098 [PMID: 15718470]

[7] Morgensztern D \& McLeod HL, AntiCancer Drugs. 2005 16: 797 [PMID: 16096426]

[8] Malavasi F et al. Physiol Rev. 2008 88: 841

[9] Johansen FE et al. Blood 2005 106: 593 [PMID: 15827133]

[10] Chaganti S et al. Blood 2009 113: 6372 [PMID: 19351961]

[11] Nelson BH, J Immunol. 2010 185: 4977 [PMID: 20962266] 
[12] Beyer M \& Schultze JL, Blood. 2006 108: 804 [PMID: 16861339]

[13] Lund FE \& Randall TD, Nat Rev Immunol. 2010 10: 236 [PMID: 20224569]

[14] Chiappelli F et al. Front Biosci. 2005 10: 3034 [PMID: 15970558]

[15] Barkhordarian A et al. Bioinformation 2011 5: 278 [PMID: 21364834]

[16] Oluwadara O et al. Bioinformation. 2009 4: 249 [PMID: 20975919]

[17] Giacomelli L et al. Bioinformation 2009 4: 258 [PMID: 20975920]
[18] Chiappelli F \& Romeo HE, Int J Oral Biol 2001 26: 39 [PMID:none]

[19] Oluwadara $\mathrm{O}$ et al. Bioinformation 2011 5: 285 [PMID: 21364836]

[20] Barkhordarian A et al. Bioinformation 2011 6: 39 [PMID: 21464844]

[21] Silvennoinen $O$ et al. J Immunol 1996 156: 100 [PMID: 8598449]

[22] Benhamron S \& Tirosh B, Eur J Immunol 2011 41: 2390 [PMID: 21674478]

[23] Barkhordarian A et al. Patholog Res Int. 2011 [PMID: 21660263]

Edited by P Kangueane

Citation: Oluwadara et al. Bioinformation 7(5): 271-275 (2011)

License statement: This is an open-access article, which permits unrestricted use, distribution, and reproduction in any medium, for non-commercial purposes, provided the original author and source are credited. 